\title{
Erratum to: Intuition and Its Object
}

\author{
Kai Hauser
}

Published online: 11 March 2015

(C) Springer Science+Business Media Dordrecht 2015

\section{Erratum to: Axiomathes \\ DOI 10.1007/s10516-014-9234-y}

In the original publication of the article, some of the references were published incorrectly. Please find below the corrected version of these references.

\section{References}

Føllesdal D (1995) Gödel and Husserl. In Hintikka J (ed) From Dedekind to Gödel: essays on the development of the foundations of mathematics. Synthese library, vol 251. Kluwer, Dordrecht 427-446

Gödel K (1947) What is Cantor's continuum problem? Am Math Mon 54:515-525 [reprinted in Gödel K (1990), pp 176-187]

Gödel K (1951) Some basic theorems on the foundations of mathematics and their implications. In: Gödel K (1995), pp 304-323

Gödel (1953/1959) Is mathematics syntax of language? In: Gödel K (1995), pp 334-362

Gödel K (1961/?) The modern development of the foundations of mathematics in the light of philosophy. In: Gödel K (1995) Transcription of shorthand draft (trans: Köhler E, Wang H, revised by Dawson J, Parsons C, Craig W), pp 374-386

Gödel K (1964) What is Cantor's continuum problem? [revised and expanded version of Gödel (1947)] In: Benacerraf P, Putnam H (eds) Philosophy of mathematics: selected readings. Cambridge University Press, Cambridge, pp 258-273 [reprinted in Gödel (1990), pp 254-270]

Gödel K (1990) Kurt Gödel collected works, vol II. In: Feferman S et al (eds). Oxford University Press, New York

The online version of the original article can be found under doi:10.1007/s10516-014-9234-y.

K. Hauser ( $\square)$

Technische Universität, Sekr. MA 5-3, 10623 Berlin, Germany

e-mail: hauser@math.tu-berlin.de 
Gödel K (1995) Kurt Gödel collected works, vol III. In: Feferman S et al (eds). Oxford University Press, New York

Gödel K (Document \#060456) Notes on the Logische Untersuchungen, contained in Gödel's Nachlass (transcribed by Dawson CA)

Heidegger (1929) Kant und das Problem der Metaphysik, 4th edn. V. Klostermann, Frankfurt a. M. (1973)

Husserl E (1887) Über den Begriff der Zahl. Psychologische Studien Habilitationschschrift, Halle [reprinted in Husserliana XII, Martinus Nijhoff, The Hague (1970), pp 289-338]

Husserl (1890-1910), Aufsätze und Rezensionen (1890-1910), Husserliana XXII. Nijhoff, The Hague, 1979

Husserl E (1891) Philosophie der Arithmetik. Logische und psychologische Untersuchungen. C. E. M. Pfeffer (Robert Stricker), Halle a. d. S. [reprinted in Husserliana XII. Martinus Nijhoff, The Hague (1970)]

Husserl E (1894) Psychologische Studien zur Elementaren Logik. Philosophische Monatshefte 30:159-191 [reprinted in Husserliana XXII, Martinus Nijhoff, The Hague (1979), pp 92-123]

Husserl E (1900) Logische Untersuchungen, Erster Teil. Prolegomena zur reinen Logik, 1. Aufl. Niemeyer, Halle

Husserl E (1901a) Logische Untersuchungen, Zweiter Band. I. Teil. Untersuchungen zur Phänomenologie und Theorie der Erkenntnis, 2. Aufl. Niemeyer, Halle (1913)

Husserl E (1901b) Logische Untersuchungen, Zweiter Band. II. Teil. Elemente einer phänomenologischen Aufklärung der Erkenntnis, 4. Aufl. Niemeyer, Tübingen (1968)

Husserl E (1907) Die Idee der Phänomenologie: Fünf Vorlesungen. Felix Meiner Verlag, Hamburg, 1986

Husserl E (1913) Ideen zu einer reinen Phänomenologie und phänomenologischen Philosophie, Erstes Buch, Husserliana III. Nijhoff, The Hague, 1950

Husserl (1925) Phänomenologische Psychologie. Felix Meiner Verlag, Hamburg, 2003

Husserl E (1927) Draft D of "phenomenology" [article subsequently published in Encyclopaedia Britannica, 14th edn, London (1929), 699-702] (trans: Palmer R). J Br Soc Phenomenol 2:77-90 (1971)

Husserl E (1929a) Formale und transzendentale Logik. Versuch einer Kritik der logischen Vernunft. Jahrbuch für Philosophie und phänomenologische Forschung 10:1-298 (English translation by Cairns D, Nijhoff, The Hague, 1969]

Husserl E (1929b) Cartesianische Meditationen. Felix Meiner Verlag, Hamburg, 1977

Husserl E (1952) Ideen zu einer reinen Phänomenologie und phänomenologischen Philosophie, Drittes Buch. In: Biemel M (ed) Husserliana V. Nijhoff, The Hague

Husserl E (1964) Erfahrung und Urteil. Untersuchungen zur Genealogie der Logik. Claassen, Hamburg Husserl E (1966) Analysen zur passiven Synthesis. Aus Vorlesungs- und Forschungsmanuskripten, 1918-1926. In: Fleischer M (ed) Husserliana XI. Nijhoff, The Hague

Locke J (1690) An essay concerning human understanding. Nidditch (ed). Oxford University Press, Oxford (1975)

Solovay RM (1965) $2^{\aleph_{0}}$ can be anything it ought to be. In: Addison JW et al (eds) The theory of models. Proceedings of the 1963 international symposium at Berkeley. North Holland, Amsterdam, p 435 